Research Paper:

\title{
Radiographic Parameters in Diagnosis of Posterior Ligamentous Complex Injury
}

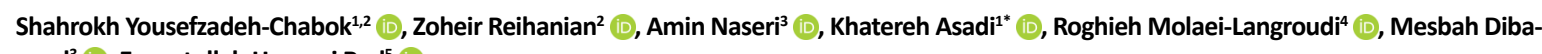
vand $^{3}$ (D), Enayatollah Homaei $\operatorname{Rad}^{5}$ (D)

1. Guilan Road Trauma Research Center, Guilan University of Medical Sciences, Rasht, Iran

2. Neuroscience Research Center, Poursina Hospital, School of Medicine, Guilan University of Medical Sciences, Rasht, Iran

3. Department of Neurosurgery, Poursina Hospital, School of Medicine, Guilan University of Medical Sciences, Rasht, Iran

4. Department of Radiology, School of Medicine, Guilan University of Medical Sciences, Rasht, Iran

5. Social Determinants of Health Research Center, Guilan University of Medical Sciences, Rasht, Iran

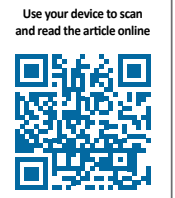

Citation: Yousefzadeh-Chabok Sh, Reihanian Z, Naseri A, Asadi Kh, Molaei-Langroudi R, \& Dibavand M, et al. Radiographic Parameters in Diagnosis of Posterior Ligamentous Complex Injury. Iran J Neurosurg. 2020; 6(3):133-142. http://dx.doi. org/10.32598/irjns.6.3.4

http://dx.doi.org/10.32598/irjns.6.3.4

Article info:

Received: 19 Mar 2020

Accepted: 13 May 2020

Available Online: $01 \mathrm{Jul} 2020$

Keywords:

CT scan imaging, Posterior Ligamentous complex, Thoracolumbar, Burst fracture

\section{ABSTRACT}

Background and Aim: Diagnosing the status of the Posterior Ligament Complex (PLC) plays an essential role in the management of patients with thoracolumbar fractures. In this study, due to the inefficiency of existing imaging modalities in the accurate detection of PLC damage, we investigated the relevance of some imaging parameters to specific guidelines for rapid PLC injury detection.

Methods and Materials/Patients: In this study, 50 patients with and 50 patients without PLC injury were included. MRI, CT scan, and radiographic imaging of the thoracolumbar spine (T12-L1) were evaluated. The thoracolumbar injury classification systems such as Denis, TLICS (Thoracolumbar Injury Classification and Scoring System), and McCormack Load Sharing and radiographic parameters such as Superior Inferior Endplate Angle (SIEA), Body Height (BH), Local Kyphosis (LK), Interspinous Distance (ISD), and Interpedicular Distance (IPD) were investigated in these patients for each imaging method. Statistical analysis was performed using SPSS V. 21 software.

Results: The ISD and LK and BHp (Body Height Posterior) were significant predictors of Posterior Ligamentous Complex (PLC) injury. On radiographs, the mean LK with and without PLC damage was $25.67^{\circ}$ and $20.92^{\circ}$, respectively $(P<0.001)$. The ISD difference was $6.75 \mathrm{~mm}$ in cases with PLC damage and $2.84 \mathrm{~mm}$ in cases with an intact PLC $(P<0.0001)$. In CT images, the mean LK was $25.77^{\circ}$ in cases with PLC damage and $18.63^{\circ}$ in cases with an intact $P L C(P<0.037)$. The ISD difference was $4.14 \mathrm{~mm}$ in patients with PLC damage and $2.19 \mathrm{~mm}$ in patients without PLC damage $(P<0.002)$. The BHp difference was $9.44 \mathrm{~mm}$ in cases with PLC damage and $11.09 \mathrm{~mm}$ in cases without PLC damage $(\mathrm{P}<0.002)$

Conclusion: The current study suggests formulating a predictive radiological index to identify PLC injury successfully. These guidelines can be very helpful in emergency room decision-makings, especially when the cost, availability, and time of performing MRI are important concerns in patients with multiple trauma.

\section{${ }^{*}$ Corresponding Author:}

Khatereh Asadi, PhD. Candidate

Address: Guilan Road Trauma Research Center, Guilan University of Medical Sciences, Rasht, Iran

Tel: +98 (13) 333368773

E-mail: khatereh70762@yahoo.com 


\section{Highlights}

- The current study suggests formulating a predictive radiological index to identify PLC injury successfully.

- We found that LK $>25$ degrees, ISD >4 mm parameters significantly predicted PLC injury.

- These guidelines can be very helpful in emergency room decision-makings, especially when the cost, availability, and time of performing MRI are important concerns in patients with multiple trauma.

\section{Plain Language Summary}

The thoracolumbar junction (T10-L2) is the most common fractured region in spinal injury. The overall prevalence of thoracolumbar fractures is higher in men, more frequent between the ages of 18-49 years old. There are various classification systems for evaluating thoracolumbar fractures, nonetheless, there is a lot of emphasis on new systems such as the Thoracolumbar Injury Classification and Scoring System (TLICS) and the McCormack Load Sharing Classification (LSC), as well as on the role of the posterior ligament complex in vertebral stability. The precise determination of the extent of posterior ligament complex injury is a key factor in the management of these patients. Radiologic parameters are an important principle for the diagnosis, classification, prognosis, spine stability, and finding the optimal treatment (conservative or surgical) which are currently interpreted based on the spine surgeon experience. Rapid assessment and medical treatment of thoracolumbar injuries can significantly improve the treatment outcomes. The present study used CT scans and plain radiography images to assess whether radiographic parameters could define PLC injury. The findings of this study suggested that the ISD and LK and BHp are significant predictors of PLC injury and can be an applicable diagnostic guideline for unstable thoracolumbar fractures.

\section{Introduction}

he thoracolumbar junction (T10-L2) is 5 the most common fractured region in spinal injury. This junction endures the skeletal load under high pressure. Increased spinal mobility in the thoracolumbar zone makes it highly vulnerable. Thoracolumbar fractures comprise more than half of spinal fractures. The annual incidence of thoracolumbar fractures has been reported 30-60 per 100,000 inhabitants $[1,2]$. The overall prevalence of thoracolumbar fractures is higher in men, more frequent between the ages of 18-49 years old [3]. Generally, about $50 \%$ of thoracolumbar fractures are unstable and are associated with neurological deficits and deformity in these patients [4]. Furthermore, thoracolumbar fractures often occur as a result of high-energy impacts such as traffic accidents and falling from a height.

The most frequent type of thoracolumbar fractures is burst fractures. They are primarily considered more severe than other fracture types which help to accurately predict spinal instability and deformity and neurological deficits $[5,6]$. There are various classification systems for evaluating thoracolumbar fractures, nonetheless, there is a lot of emphasis on new systems such as the Thoracolumbar Injury Classification and Scoring System (TLICS) and the McCormack Load Sharing Classification (LSC), and on the role of the posterior ligament complex in vertebral stability $[7,8]$.

The posterior ligament complex consists of structures such as supraspinous ligaments, interspinous ligamentum, articular capsule, and ligamentum flavum. These ligaments play a supporting role in the resistance and stability of the vertebral column. Nevertheless, accurate detection of the severity of Posterior Ligamentous Complex (PLC) injury remains dubious [9-11]. Primarily, the diagnosis of unstable thoracolumbar fractures is very critical for prescribing the appropriate treatment (surgical or non-surgical) which is required to prevent vertebral column deformity and secondary neural deficit. Therefore, the precise determination of the extent of posterior ligament complex injury is a key factor in the management of these patients.

Radiologic parameters are an important principle for the diagnosis, classification, prognosis, spine stability, and finding the optimal treatment (conservative or surgical) which are currently interpreted based on the spine surgeon experience. Radiological imaging is very 
important in determining the segment of vertebral fractures. Although the Multidetector Computed Tomography scan (MDCT), quick and accurate in patients without neurologic deficits, has high sensitivity and acceptable specificity for effective screening of vertebral fracture type, generally, CT has low efficacy in diagnosing soft tissue injuries. Therefore, using a radiographic examination coupled with CT scan can improve the detection of soft tissue injury [12-15]. Some studies have shown that MRI has a high sensitivity to PLC injury detection, but interrater reliability using Short-TI Inversion Recovery (STIR) was only $70 \%$ and was much lower (48.9\%) using sagittal T1 magnetic resonance imaging (MRI) which can be seen as enhanced signals on the T2 images and diagnosed as unstable fracture patterns [16]. Thus, it can be realized that MRI is suitable for detecting bony edema which can be a symptom of an occult fracture contributing to preoperative considerations on levels of fixation [17].

Furthermore, for early diagnosis of spinal fracture, MRI has been controversial as a result of limited accessibility to MRI examinations which not only impose considerably high economic costs on the healthcare system but also expose patients with multiple trauma to imaging challenges [18-20]. Therefore, there are no certain radiological criteria and CT scans to determine PLC injury. The number of reports on the efficacy of this matter is limited and the aim of this study is not to use MRI for PLC injury clinically. Variable radiological and CT scan parameters for PLC disorder detection have been reported in the literature and publications on the thoracolumbar spine. However, none of these parameters are considered as a guideline [21]. This study aimed to evaluate the extent of PLC damages by some important radiological parameters including SIEA, $\mathrm{BH}, \mathrm{LK}, \mathrm{ISD}$, and IPD concerning CT scan and radiological findings to determine their value for the rapid and optimal diagnosis and treatment of thoracolumbar fractures.

\section{Methods and Materials/Patients}

This study was performed retrospectively on patients with thoracolumbar spinal fractures without neurological deficit who had referred to Poursina Teaching Hospital in Rasht (North of Iran) from April 2016 to March 2018 which was approved by the institutional etic board (IR.GUMS.REC.1398.065). Patients with fractures in the thoracolumbar junction between T10 and L2 and the diagnosis of burst or compression fractures, with and without Posterior Ligament Complex (PLC) injury were included in the study. The minimum inclusion age based on the time of growth plate closure was 25 years and the maximum age of inclusion was 65 years, based on bone tissue density. All of the patients underwent radiographic $\mathrm{CT}$, and MRI imaging. Radiographic images were taken from the thoracolumbar region of the dorsal and lumbar vertebrae in lateral and anterior-posterior views. Patients were assessed with $\mathrm{CT}$ in axial view which was then reconstructed to the sagittal plane according to the protocols in the device. These images were prepared from a single CT scan scanner (16 Slice, Supria; Hitachi Medical Corporation, Japan).

We evaluated the established plain radiographs and mid-sagittal CT scans based on the following parameters (Figure 1):

1. Superior Inferior Endplate Angle (SIEA): the measured angle in the upper and lower extremities of the fractured segment

2. Body Height $(\mathrm{BH})$ : This factor is calculated as a percentage of the reduction of the height of the vertebral body relative to the average of the upper and lower vertebral bodies of the affected area.

3. The local kyphosis angle is calculated as the angle between the upper and lower extremities of the damaged segment.

4. Interspinous Distance (ISD): this factor is calculated from the middle region of the cephalad surface of the injured vertebrae's spinous process relative to the middle part of the cauda of the cephalad surface of the spinous process.

5. Interpedicular Distance (IPD): this parameter was measured as the distance between the interlines of the two fractured vertebral pedicels. This distance is compared to the average size of the healthy vertebrae in the cephalad and caudal segments of the fractured area.

MRI images were taken in 3 T1-weighted, T2-weighted, and STIR protocols from a single 1 Tesla MRI scanner (Gyroscan, Philips Medical Systems, Germany). The STIR protocol was used in the sagittal plane to evaluate the damage to the posterior ligament complex and the extent of its damage was assessed by grading (intact, injured) in all patients. The integrity of the PLC in TLICS systems is categorized as intact, indeterminate disruption, or clear disruption. The most reliable signs of ligamentous injury are disruption of the black stripe denoting low signal intensity on sagittal T1- or T2-weighted $\mathrm{MRI}$, indicating a supraspinous ligament or ligamentum flavum tear. In addition, areas of high signal intensity within facet capsules or interspinous region on fluid- 
sensitive STIR or T2-weighted MRI also reflect capsular or interspinous ligament injury. MRI was required for all patients in order to determine the TLICS score and define the type of conservative or surgical treatment. At the final stage, the statistical analysis was performed. The parameters measured in radiographic, $\mathrm{CT}$, and $\mathrm{MRI}$ images were evaluated in both groups of patients with and without PLC injury. Ultimately, the statistical differences between the parameters measured in the groups with intact PLC and with damaged PLC were evaluated and compared. Sensitivity and specificity were calculated for this purpose, ROC (Receiver operating characteristic curve) curves were plotted using Stata 13.1 version (StataCorp, College Station, Texas 77845 USA).

\section{Result}

In this study, 50 patients with and 50 patients without PLC injury were included. We showed the patients' demographic data and fracture features in Table 1.

\section{The analysis of parameters in radiographic images}

SIEA, ISD, IPD, LK, BHp, and BHA parameters assessed in plain radiography images that $L K, I S D$, and $B H p$ values were significant $(P<0.05)$. The mean ISD in the PLC injured group was $6.75 \mathrm{~mm}$ when compared to PLC intact group, the mean ISD was $2.84 \mathrm{~mm}(P<0.0001)$. The mean of $L K$ in the PLC injured group was $25.70^{\circ}$ while that of the PLC intact group was $20.92^{\circ}(P<0.001)$. The mean $\mathrm{BHp}$ in plain radiographic images was $11.54 \mathrm{~mm}$ in the PLC injured group and $15.20 \mathrm{~mm}$ in the PLC intact group $(\mathrm{P}<0.05)$ (Table 2$)$.

\section{Parameters of analysis in CT scan images}

SIEA, ISD, IPD, LK, BHp, and BHA were evaluated in CT images in which LK, ISD, and $\mathrm{BHp}$ values were significant $(P<0.05)$. The Mean \pm SD of ISD in the injured PLC group was about $4.14 \pm 1.3$ while in the intact PLC group, It was $2.19 \pm 1.2$ with $P=0.02$. The Mean $\pm S D$ values of $L K$ in the injured PLC group were about $25.80 \pm 4.03$ while that of the intact PLC group was about $18.63 \pm 3.1$ with $\mathrm{P}=0.001$. The Mean $\pm S D$ values of BHP in CT images in the injured $\mathrm{PLC}$ group was $9.43 \pm 2.9$ and in the PLC intact group was $11.09 \pm 5.3$ with a significance level of $P=0.048$ (Table 3).

Receiver operating characteristic (ROC) curve graphs (Figure 2) display the sensitivity and specificity of CT scan and radiographic in PLC injury detection based on the local kyphotic deformity, interspinous distance, and posterior vertebral body height [22]. Sensitivity and specificity indices for identifying PLC injury were evaluated for each of the LK and ISD parameters in radiographic images. The 20 degrees LK had a sensitivity of $48.25 \%$ and a specificity of $56.16 \%$ to determine the PLC damage. When LK increased to 25 degrees, the sensitivity and specificity increase was $63.51 \%$ and $88.46 \%$, respectively. When ISD was considered as a single factor in detecting PLC damage, the $2 \mathrm{~mm}$ ISD had a sensitivity of $46.28 \%$ and a specificity of $53.25 \%$; though when ISD increased to $4 \mathrm{~mm}$, the sensitivity and specificity were $51.9 \%$ and $72.86 \%$, respectively. Considering the two factors, ISD increased by more than $4 \mathrm{~mm}$ and LK by more than 25 degrees; sensitivity and specificity were $68.67 \% 96.01 \%$, respectively.
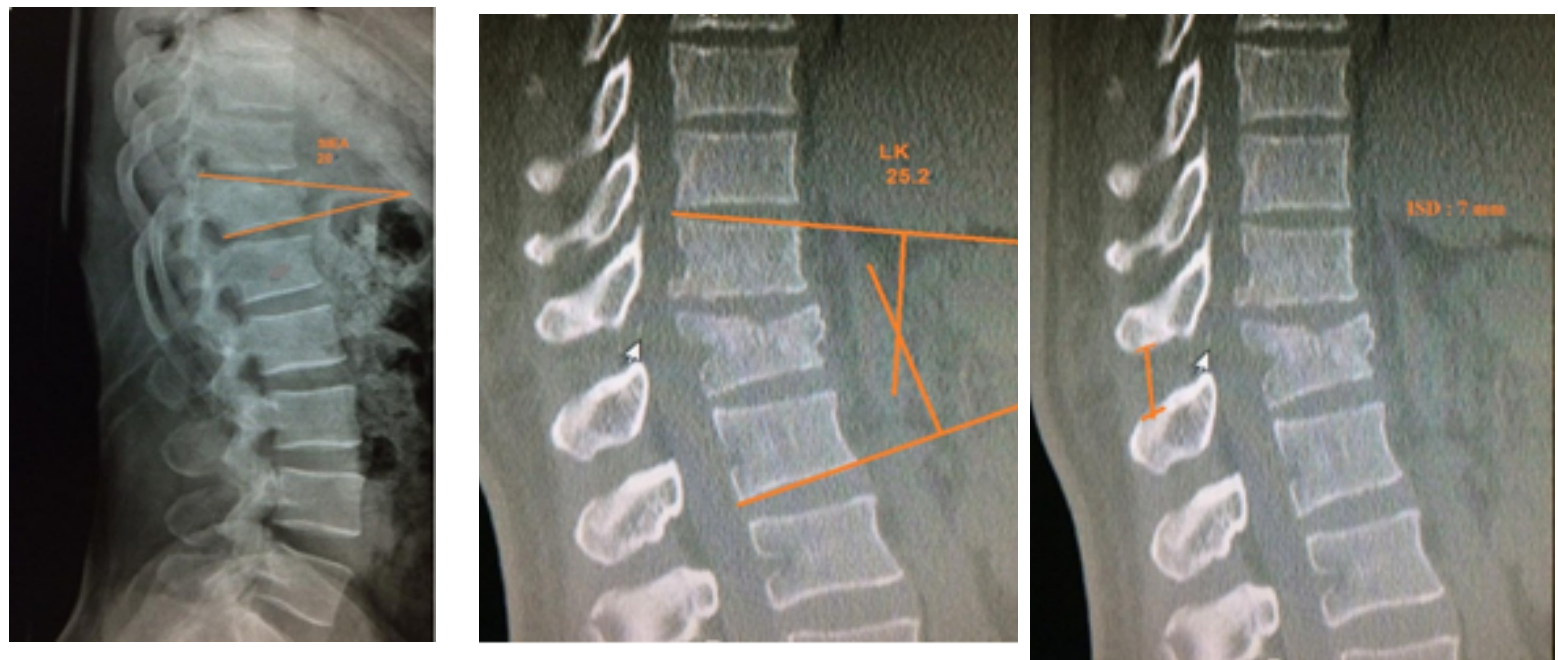

Figure 1. The radiographic images

A: Measurement of Superior-Inferior Endplate Angle (SIEA) in lateral plain film radiography; B: Measurement of Local Kyphosis angle (LK); C: Interspinous Distance (ISD) in midsagittal 
Table 1. Patient characteristics in this study

\begin{tabular}{|c|c|c|}
\hline \multicolumn{2}{|c|}{ Patient Characteristics } & \multirow{2}{*}{$\begin{array}{c}\text { Frequency } \\
100 \text { (50 PLC injured, 50 PLC intact) }\end{array}$} \\
\hline Number of cases & Total & \\
\hline \multirow{3}{*}{ Gender } & Women & 52 (25\% PLC injured, 23\% PLC intact) \\
\hline & & \\
\hline & Men & 48 (25\% PLC injured, 27\% PLC intact) \\
\hline \multirow[t]{3}{*}{ Mean $\pm S D$ age $(y)$} & \multicolumn{2}{|c|}{$37.99 \pm 11.59$ (38.42 \pm 11.68 PLC injured, $37.56 \pm 11.60$ PLC intact) } \\
\hline & $\mathrm{T} 11$ & 27 (13 PLC injured, 14 PLC intact) \\
\hline & T12 & 26 (11 PLC injured, 15 PLC intact) \\
\hline \multirow[t]{3}{*}{ Fracture site } & L1 & 23 (12 PLC injured, 11 PLC intact) \\
\hline & $\mathrm{L} 2$ & 24 (13 PLC injured, 11 PLC intact) \\
\hline & Total of TLICS $\pm S D$, points & 3.11 \pm 0.78 (3.72 \pm 1.24 PLC injured, $2.48 \pm 0.61$ PLC intact) \\
\hline
\end{tabular}

Sensitivity and specificity indices were evaluated for each of the LK and ISD parameters in CT scans. The LK value of 20 degrees had a sensitivity of $55.8 \%$ and a specificity of $58.52 \%$ to determine the extent of PLC damage. Whereas with the increase of LK by 25 degrees, the sensitivity increased by $62.25 \%$ and specificity by $94.11 \%$. When ISD is considered as a single factor in detecting PLC damage, the $2 \mathrm{~mm}$ ISD has a sensitivity of $50.01 \%$ and a specificity of $58.21 \%$ which ISD increases to $4 \mathrm{~mm}$, respectively the sensitivity and specificity were $70.58 \%$ and $96.65 \%$. Considering the 2 factors ISD increased by more than $4 \mathrm{~mm}$ and LK by more than 25 degrees sensitivity and specificity were $70.8 \%$ and $97.52 \%$. Confidence Interval $(\mathrm{Cl})$ was assessed on plain radiographs and $\mathrm{CT}$ scan images. The presence of $\mathrm{LK}$ greater than $25^{\circ}$ (Cl 64\%-95\%), ISD difference greater

Table 2. Results of the assessed variables on plain radiographs

\begin{tabular}{|c|c|c|c|}
\hline Measurement RAD & PLC & Mean $\pm S D$ & $\mathbf{P}$ \\
\hline \multirow{3}{*}{$\mathrm{ISD}(\mathrm{mm})$} & Injury & $6.74 \pm 2.90$ & \multirow{3}{*}{$0.0001 * *$} \\
\hline & & & \\
\hline & Intact & $2.83 \pm 1.25$ & \\
\hline \multirow{3}{*}{$\operatorname{IPD}(\mathrm{mm})$} & Injury & $19.19 \pm 9.13$ & \multirow{3}{*}{0.745} \\
\hline & & & \\
\hline & Intact & $18.62 \pm 9.17$ & \\
\hline \multirow{3}{*}{ LK deformity angle (degrees) } & Injury & $25.69 \pm 4.41$ & \multirow{3}{*}{$0.001^{* *}$} \\
\hline & & & \\
\hline & Intact & $20.92 \pm 3.11$ & \\
\hline \multirow{3}{*}{ SIEA angle (degrees) } & Injury & $22.85 \pm 5.33$ & \multirow{3}{*}{0.155} \\
\hline & & & \\
\hline & Intact & $18.24 \pm 4.84$ & \\
\hline \multirow{3}{*}{$\mathrm{BH}$ anterior $(\mathrm{mm})$} & Injury & $48.85 \pm 8.12$ & \multirow{3}{*}{0.365} \\
\hline & & & \\
\hline & Intact & $53.35 \pm 7.60$ & \\
\hline \multirow{3}{*}{ BH posterior (mm) } & Injury & $11.54 \pm 5.58$ & \multirow{3}{*}{$0.048^{*}$} \\
\hline & & & \\
\hline & Intact & $15.20 \pm 6.88$ & \\
\hline
\end{tabular}


Table 3. Results for the variables when assessed on CT scan

\begin{tabular}{cccc}
\hline Measurement & PLC & Mean \pm SD & P \\
\hline ISD $(\mathrm{mm})$ & Injury & $4.14 \pm 1.30$ & $0.020^{*}$ \\
\hline IPD $(\mathrm{mm})$ & Intact & $2.19 \pm 1.20$ & 0.261 \\
& Injury & $17.73 \pm 7.22$ & $0.037^{*}$ \\
\hline LK deformity angle (degrees) & Intact & $18.14 \pm 6.64$ & 0.243 \\
\hline IEA angle (degrees) & Injury & $25.79 \pm 4.03$ & 0.464 \\
\hline BH anterior $(\mathrm{mm})$ & Intact & $18.63 \pm 3.12$ & $17.49 \pm 4.45$ \\
& Injury & $14.22 \pm 4.93$ & $0.0001^{* *}$ \\
\hline
\end{tabular}

${ }^{*} \mathrm{P}<0.05 ;{ }^{* * P}<0.01$

than $4 \mathrm{~mm}(\mathrm{Cl} 70 \%-97 \%)$, and $\mathrm{BHp}$ lower than $9 \mathrm{~mm} \mathrm{(Cl}$ $60 \%-76 \%)$ could predict PLC injury.

\section{Discussion}

The present study used CT scan and plain radiography images to assess whether radiographic parameters could define PLC injury. The findings of this study suggested that the ISD and LK and BHp to be significant predictors of PLC injury and can be an applicable diagnostic guideline for unstable thoracolumbar fractures. Rapid assessment and medical treatment of thoracolumbar injuries can significantly improve the treatment outcomes [23, 24]. So far, different classification systems have been developed to assess the thoracolumbar spinal injuries such as TLICS, determined through neurological examinations and diagnostic modalities such as plain radiography, CT scans, and MRIs while the physician requires the simplest criteria to get the best decision in the shortest time concerning high patient volume. PLC injury extension plays an important role in determining the effective treatment for burst fractures. PLC has a great supporting role in vertebral stability and strength. It has a poorly-healed tissue that is mainly due to weak vasculature. PLC disruptions indicate damages to its components i.e. supraspinous Ligament (SSL), Interspinous Ligament (ISL), Facet Capsule Ligament (FCL), and Ligamentum Flavum (LF) and integrating units such as fascia and musculature. PLC injuries may lead to spinal instability, often coupled with noticeable spinal column deformity and neurological deficit. Recent studies suggest that in the majority of cases, MRI tends to overdiagnose PLC injury that would lead to an increase in unnecessary surgeries $[25,26]$.

In a survey conducted to determine the diagnostic accuracy of MRI in PLC injuries, despite the previously reported sensitivity up to 100 , the accuracy of MRI to diagnose PLC injuries was low, so it should not be used alone for detecting PLC integrity, for choosing the most effective treatment for thoracolumbar fractures and most important of all, for differentiating self-healing disruptions from those needing to be surgically fixed to prevent a further collapse [27].

In another study, Pizones et al. reported that MRI played an important role in screening PLC injuries but when using MRI alone, it is difficult to identify and measure the extent and type of PLC damage. STIR and FS-T2 sequences can help detect connective tissue injuries. However, the severity of PLC damage and differentiating self-healing PLC disruptions from the ones requiring surgical fixation to prevent a further collapse are still debatable $[19,28]$ 

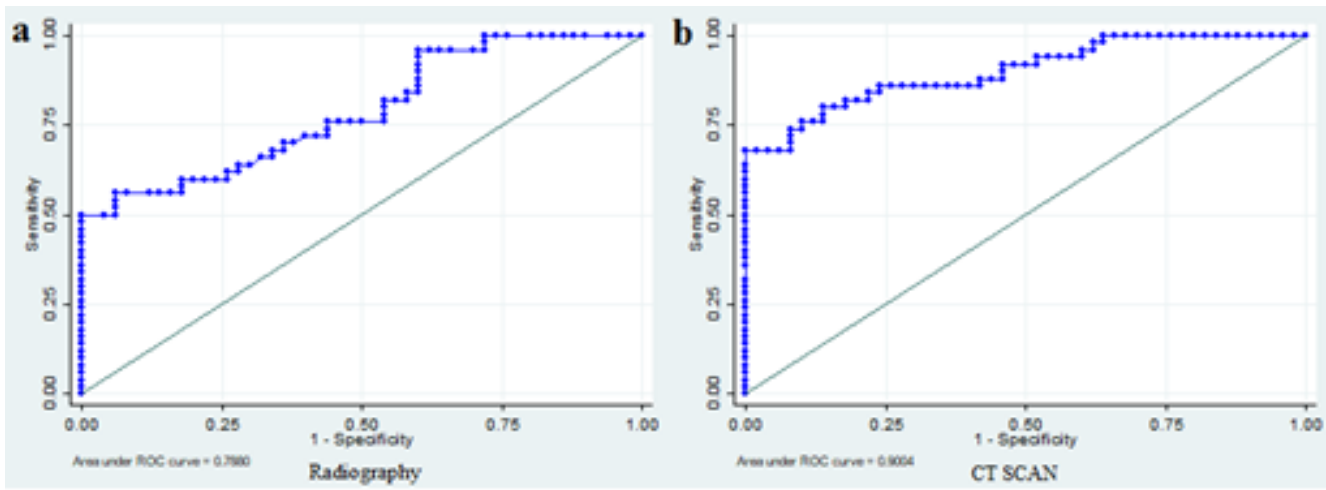

Local Kyphotic Deformity Ranges in Degrees
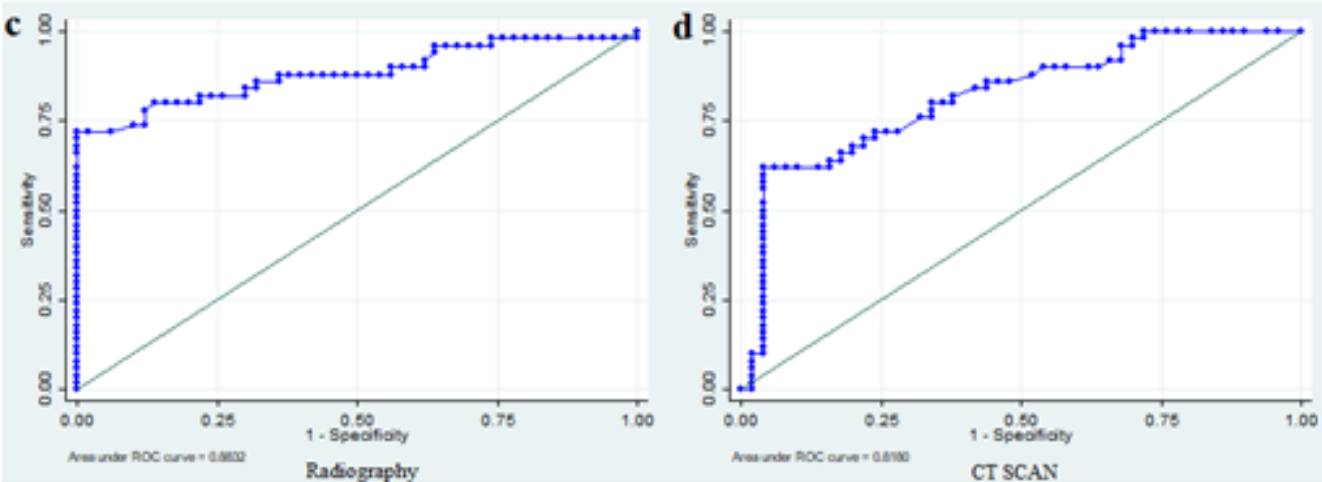

Interspinous Distance Ranges in Millimeters
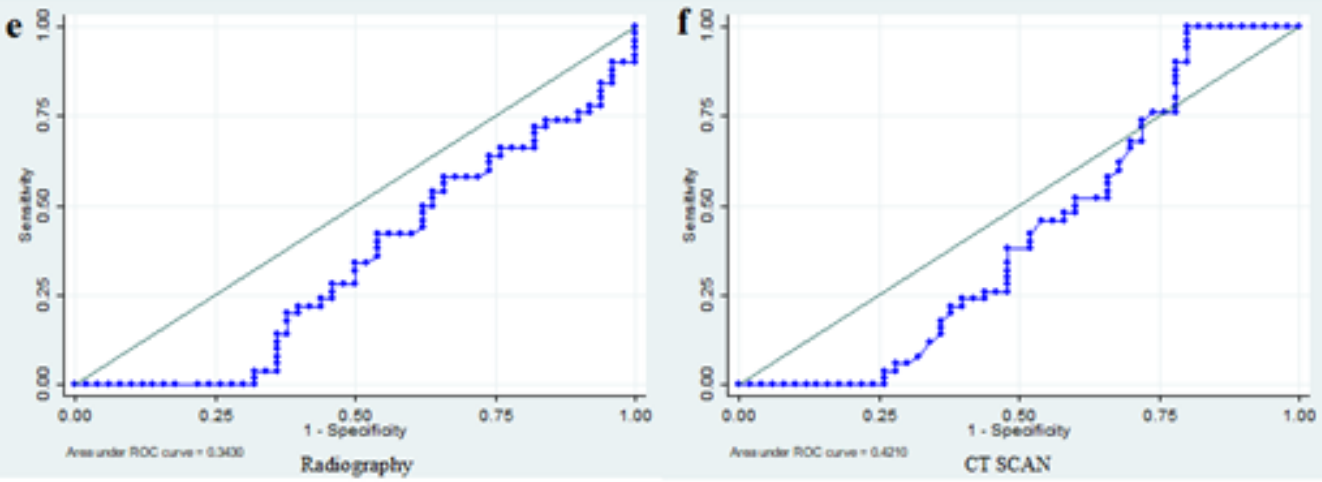

Body Hight Posterior Ranges in Millimeters

Figure 2. Receiver Operating Characteristic (ROC) curve graphs

A, C, E: ROC curve of local kyphotic deformity (degree), interspinous distance (millimeters), and posterior vertebral body height in radiographic images; B, D, F: ROC curve of local kyphotic deformity, interspinous distance, and posterior vertebral body height in CT scan images

The ROC graph of CT scan images determined radiographic results. The graph presents the values of PLC injury of thoracolumbar junction within the mentioned parameters. The curve shows an increase in the percentage of $P L C$ injuries beyond $25^{\circ}$ of $L K, 4 \mathrm{~mm} \mathrm{ISD}$, and $9 \mathrm{~cm} \mathrm{BHp}$.

Hiyama et al. in a survey assessed the accuracy of PLC injury in thoracolumbar burst fracture determined by STIR sequence using radiologic parameters and showed that LOVBH (Loss of Vertebral Body Height), vertebral body translation, and canal compromise do not correlate significantly with the presence of a PLC injury in patients with thoracolumbar fractures, an $\mathrm{LK}>20^{\circ}$ and increased the percentage of SSD (Supraspinous Distance) are as- sociated with a PLC injury. This study insisted on the fundamental role of diagnostic criteria and classification systems in determining PLC damage and prescribing effective therapeutic procedures. The TLICS classification is based on MRI findings which is sensitive to detecting PLC injuries. However, it is more specific to determine the type of fractures (burst, compression, etc.) than in comparison to plain radiography and CT scans [23]. 
Currently, CT imaging besides plain radiography is being investigated as one of the effective examinations in the diagnosis of the spinal cord and PLC injuries [17]. Rajasekaran et al. performed a study on predicting PLC injuries in thoracolumbar fractures based on radiographic imaging and CT scans without MRI. They assessed 5 radiographic parameters in plain radiography and CT scan in which LK and ISD between injured and intact groups were significant $(P<0.05)$. Sensitivity in the LK above 20 degrees was about $76 \%$ for CT scans and $85 \%$ for plain radiographs. If the ISD is increased to $4 \mathrm{~mm}$, the specificity for CT and plain radiography is more than $90 \%$ [2].

In this study, after analyzing the results, we found that LK, ISD, BHp parameters were significant in predicting PLC injury between PLC-injured and intact groups $(P<0.05)$. The specificity value was $58.12 \%$ for LK more than 20 degrees in CT scan and $56.16 \%$ in radiography, which increased over $88 \%$ in LK greater than 25 degrees. It seems that the increase in LK is caused by high-energy trauma leading to spine instability that plays an important role in choosing the treatment modality (conservative or surgical). Thus, measuring the change in the kyphotic angle has a limitation as a single test for the diagnosis of the injury in the posterior ligament complex [29-31].

Vaccaro et al. claimed that interspinous spacing greater than that of the abovementioned level or below on anteroposterior plain X-rays can be higher rank criteria for the diagnosis of PLC injury than focal tenderness and palpation of the interspinous defect. This study showed that by increasing the ISD parameter more than $2 \mathrm{~mm}$, the sensitivity and specificity in detecting PLC injury in CT scan augmented to $50.01 \%$ and $58.21 \%$, respectively, and in radiology augmented to 46.28 and 53.25 between the injured and intact groups. ISD more than 4 $\mathrm{mm}$ also increases sensitivity to over $70 \%$ and specificity to greater than $90 \%$. According to the result, if ISD increases, the incidence of PLC injury increases. Therefore, it can be considered as a significant parameter in the diagnosis of PLC injury [27, 32, 33].

The results of vertebral height measurement presented that the reduction of posterior vertebral height was significant between PLC injured and intact groups. The specificity and sensitivity of this parameter were low, which could be due to the error of the manual measurement method [34, 35].

Also by considering the two parameters, LK and ISD, the specificity and sensitivity of the results were $96.01 \%$ and $68.67 \%$ on plain radiographs and increased to more than $70.8 \%$ and $97.52 \%$ on CT scans, respectively.
Therefore, integrating several parameters can provide a more accurate and efficient diagnosis.

\section{Conclusion}

As the finding of the present study indicated, PLC injury had a significant relationship with LK, ISD, and BH. The current study suggests formulating a predictive radiological index to identify PLC injury successfully. These guidelines may be utilized in emergency room decisionmaking especially when the associated cost, availability, and time delay in performing MRI are a concern in patients with multiple trauma.

\section{Ethical Considerations}

\section{Compliance with ethical guidelines}

The study was approved by The Ethics Committee of Guilan University of Medical Sciences (Code: IR.GUMS. REC.1398.065).

\section{Funding}

This research did not receive any grant from funding agencies in the public, commercial, or non-profit sectors.

\section{Authors contributions}

Conception and design: Shahrokh YousefzadehChabok, Zoheir Reihanian, Amin Naseri; Data collection: Khatereh Asadi, Amin Naseri, Mesbah Dibavand; Data analysis and interpretation: Khatereh Asadi, Amin Naseri, Mesbah Dibavand; Drafting the article: Enayatollah Homaie Rad, Roghieh Molaei-Langroudi; Critically revising the article: Khatereh Asadi, Amin Naseri, Shahrokh Yousefzadeh-Chabok, Zoheir Reihanian; Reviewing submitted version of manuscript: Khatereh Asadi, Amin Naseri, Shahrokh Yousefzadeh-Chabok; Approving the final version of the manuscript: All authors.

\section{Conflict of interest}

The authors declared no conflict of interest.

\section{Acknowledgements}

The authors would like to offer their special thanks to Ms.Fatemeh Javadi for proofreading the language of the manuscript. 


\section{References}

[1] Sadiqi S, Dvorak MF, Vaccaro AR, Schroeder GD, Post MW Benneker LM, et al. Reliability and validity of the English version of the AOSpine PROST (Patient Reported Outcome Spine Trauma). Spine. 2020; 45(17):E1111-8. [DOI:10.1097/ BRS.0000000000003514] [PMID] [PMCID]

[2] Rajasekaran Sh, Maheswaran A, Aiyer SN, Kanna R, Dumpa SR, Shetty AP. Prediction of posterior ligamentous complex injury in thoracolumbar fractures using non-MRI imaging techniques. International Orthopaedics. 2016; 40(6):1075-81. [DOI:10.1007/s00264-016-3151-1] [PMID]

[3] Farr JN, Joseph Melton III L, Achenbach SJ, Atkinson EJ, Khosla S, Amin Sh. Fracture incidence and characteristics in young adults aged 18 to 49 years: A population-based study. Journal of Bone and Mineral Research . 2017; 32(12):2347-54 [DOI:10.1002/jbmr.3228] [PMID] [PMCID]

[4] Rajasekaran S, Kanna RM, Shetty AP. Management of thoracolumbar spine trauma: An overview. Indian Journal of Orthopaedics. 2015; 49(1):72-82. [DOI:10.4103/00195413.143914] [PMID] [PMCID]

[5] Heinzelmann M, Wanner GA. Thoracolumbar spinal injuries.In: Boos N, Aebi M, editors. Spinal Disorders. Berlin/ Heidelberg: Springer; 2008. pp. 883-924. [DOI:10.1007/978-3540-69091-7_31]

[6] Katsuura Y, Osborn JM, Cason GW. The epidemiology of thoracolumbar trauma: A meta-analysis. Journal of Orthopaedics. 2016; 13(4):383-8. [DOI:10.1016/j.jor.2016.06.019] [PMID] [PMCID]

[7] Gomleksiz C, Egemen E, Senturk S, Yaman O, Aydın AL, Oktenoglu $\mathrm{T}$, et al. Thoracolumbar fractures: A review of classifications and surgical methods. Journal of Spine. 2015; 4(4):1000250. [DOI:10.4172/2165-7939.1000250]

[8] Joaquim AF, de Almeida Bastos DC, Jorge Torres $\mathrm{HH}$, Patel AA. Thoracolumbar injury classification and injury severity score system: A literature review of its safety. Global Spine Journal. 2016; 6(1):80-5. [DOI:10.1055/s-0035-1554775] [PMID] [PMCID]

[9] Azam MQ, Sadat-Ali M. The concept of evolution of thoracolumbar fracture classifications helps in surgical decisions. Asian Spine Journal. 2015; 9(6):984-94. [DOI:10.4184/ asj.2015.9.6.984] [PMID] [PMCID]

[10] Aebi M. Classification of thoracolumbar fractures and dislocations. European Spine Journal. 2010; 19 Suppl 1(Suppl 1):2-7. [DOI:10.1007/s00586-009-1114-6] [PMID] [PMCID]

[11] Atlas SW, Regenbogen V, Rogers LF, Kim KS. The radiographic characterization of burst fractures of the spine. American Journal of Roentgenology. 1986; 147(3):575-82 [DOI:10.2214/ajr.147.3.575] [PMID]

[12] Defino HLA, Canto FRT. Low thoracic and lumbar burst fractures: Radiographic and functional outcomes. European Spine Journal. 2007; 16(11):1934-43. [DOI:10.1007/ s00586-0070406-y] [PMID] [PMCID]

[13] Vaccaro AR, Kim DH, Brodke DS, Harris M, Chapman JR, Schildhauer T, et al. Diagnosis and management of thoracolumbar spine fractures. Instructional Course Lectures. 2004; 53:359-73. [PMID]
[14] Burns JE, Yao J, Muñoz H, Summers RM. Automated detection, localization, and classification of traumatic vertebra body fractures in the thoracic and lumbar spine at CT. Radiology. 2015; 278(1):64-73. [DOI:10.1148/radiol.2015142346] [PMID] [PMCID]

[15] Schwartz ED, Flanders AE. Spinal trauma: Imaging, diagnosis, and management. American Journal of Neuroradiology. 2008; 29(5):e37. [DOI:10.3174/ajnr.A0978]

[16] Harrop JS, Vaccaro AR, Hurlbert RJ, Wilsey JT, Baron EM Shaffrey CI, et al. Intrarater and interrater reliability and validity in the assessment of the mechanism of injury and integrity of the posterior ligamentous complex: A novel injury severity scoring system for thoracolumbar injuries. Journal of Neurosurgery Spine. 2006; 4(2):118-22. [DOI:10.3171/ spi.2006.4.2.118] [PMID]

[17] Vaccaro AR, Su BW, Chiba K, Dvorak MF, Mayer HM, Rajasekaran S, et al. The spine: Medical \& surgical management: Two volume set. New Delhi: Jaypee Brothers Medical Publishers; 2019. https://books.google.com/ books?id=13KSDwAAQBAJ\&dq

[18] Santiago FR, Muñoz PT, Sánchez EM, Paniza MR, Martínez AM, Abela ALP. Classifying thoracolumbar fractures: Role of quantitative imaging. Quantitative imaging in medicine and surgery. 2016; 6(6):772-84. [DOI:10.21037/ qims.2016.12.04] [PMID] [PMCID]

[19] Pizones J, Izquierdo E, Álvarez P, Sánchez-Mariscal F, Zúñiga $\mathrm{L}$, Chimeno $\mathrm{P}$, et al. Impact of magnetic resonance imaging on decision making for thoracolumbar traumatic fracture diagnosis and treatment. European Spine Journal. 2011; 20 Suppl 3(Suppl 3):390. [DOI:10.1007/s00586-0111913-4] [PMID] [PMCID]

[20] Öner FC, Wood KB, Smith JS, Shaffrey CI. Therapeutic decision making in thoracolumbar spine trauma. Spine 2010; 35(21S):S235-44. [DOI:10.1097/BRS.0b013e3181f32734] [PMID]

[21] Vaccaro AR, Lee JY, Schweitzer Jr KM, Lim MR, Baron EM, Öner FC, et al. Assessment of injury to the posterior ligamentous complex in thoracolumbar spine trauma. The Spine Journal. 2006; 6(5):524-8. [DOI:10.1016/j. spinee.2006.01.017] [PMID]

[22] Hajian-Tilaki K. Receiver Operating Characteristic (ROC) curve analysis for medical diagnostic test evaluation. Caspian Journal of Internal Medicine. 2013; 4(2):627-35. http:// caspjim.com/article-1-193-en.html

[23] Hiyama A, Watanabe M, Katoh H, Sato M, Nagai T, Mochida J. Relationships between posterior ligamentous complex injury and radiographic parameters in patients with thoracolumbar burst fractures. Injury. 2015; 46(2):392-8. [DOI:10.1016/j.injury.2014.10.047] [PMID]

[24] Leucht P, Fischer K, Muhr G, Mueller EJ. Epidemiology of traumatic spine fractures. Injury. 2009; 40(2):166-72 [DOI:10.1016/j.injury.2008.06.040] [PMID]

[25] Zeng J, Gong Q, Liu H, Rong X, Ding Ch. Complete fracture-dislocation of the thoracolumbar spine without neurological deficit: A case report and review of the literature. Medicine. 2018; 97(9):e0050. [DOI:10.1097/ MD.0000000000010050] [PMID] [PMCID]

[26] Machino M, Yukawa Y, Ito K, Kanbara Sh, Morita D, Kato F. Posterior ligamentous complex injuries are related to frac- 
ture severity and neurological damage in patients with acute thoracic and lumbar burst fractures. Yonsei Medical Journal. 2013; 54(4):1020-5. [DOI:10.3349/ymj.2013.54.4.1020] [PMID] [PMCID]

[27] Vaccaro AR, Rihn JA, Saravanja D, Anderson DG, Hilibrand AS, Albert TJ, et al. Injury of the posterior ligamentous complex of the thoracolumbar spine: A prospective evaluation of the diagnostic accuracy of magnetic resonance imaging. Spine. 2009; 34(23):E841-7. [DOI:10.1097/ BRS.0b013e3181bd11be] [PMID]

[28] Lee HM, Kim HS, Kim DJ, Suk KS, Park JO, Kim $\mathrm{NH}$. Reliability of magnetic resonance imaging in detecting posterior ligament complex injury in thoracolumbar spinal fractures. Spine. 2000; 25(16):2079-84 [DOI:10.1097/00007632-200008150-00012] [PMID]

[29] Shen WJ, Liu TJ, Shen YS. Nonoperative treatment versus posterior fixation for thoracolumbar junction burst fractures without neurologic deficit. Spine. 2001; 26(9):1038-45. [DOI:10.1097/00007632-200105010-00010] [PMID]

[30] Curfs I, Grimm B, van der Linde M, Willems P, van Hemert W. Radiological prediction of posttraumatic kyphosis after thoracolumbar fracture. The Open Orthopaedics Journal. 2016; 10:135-42. [DOI:10.2174/187432500161001013 5] [PMID] [PMCID]

[31] Hartmann F, Nusselt T, Mattyasovszky S, Maier G, Rommens PM, Gercek E. Misdiagnosis of thoracolumbar posterior ligamentous complex injuries and use of radiographic parameter correlations to improve detection accuracy. Asian Spine Journal. 2019; 13(1):29-34. [DOI:10.31616/ asj.2017.0333] [PMID] [PMCID]

[32] Kwon KY, Park HJ, Shin JS, Lee JP. Another diagnostic tool in thoracolumbar posterior ligament complex injury: Interspinous distance ratio. European Spine Journal. 2017; 26(5):1447-53. [DOI:10.1007/s00586-016-4662-6] [PMID]

[33] Meves R, Avanzi O. Can the interspinous distance predict kyphosis in conservative treatment in thoracolumbar burst fracture? A retrospective, diagnostic study. Open Journal of Orthopedics. 2016; 6(1):10-5. [DOI:10.4236/ojo.2016.61002]

[34] Daffner RH, Deeb ZL, Rothfus WE. The posterior vertebral body line: Importance in the detection of burst fractures. American Journal of Roentgenology. 1987; 148(1):936. [DOI:10.2214/ajr.148.1.93] [PMID]

[35] Radcliff K, Su BW, Kepler CK, Rubin T, Shimer AL, Rihn JA, et al. Correlation of posterior ligamentous complex injury and neurological injury to loss of vertebral body height, kyphosis, and canal compromise. Spine. 2012; 37(13):114250. [DOI:10.1097/BRS.0b013e318240fcd3] [PMID] 